\title{
Resultados tempranos en pacientes con cáncer gástrico sometidos a gastrectomía laparoscópica con intención curativa
}

\author{
Early results in gastric cancer patients undergoing laparoscopic \\ gastrectomy with curative intent
}

\author{
Paula Andrea Jurado-Muñoz ${ }^{1} \mathbb{D}$, Robin Hernando Bustamante-Múnera' $\mathbb{D}^{\mathbb{D}}$, \\ Juan Pablo Toro-Vásquez ${ }^{2} \mathbb{D}$, Juan Camilo Correa-Cote ${ }^{3} \mathbb{D}$, Carlos Hernando Morales-Uribe $\mathbb{D}^{4}$
}

\begin{abstract}
Médico, residente de Cirugía general, Universidad de Antioquia, Medellín, Colombia.
Médico, especialista en Cirugía general y Cirugía mínimamente invasiva; cirujano asistencial, Hospital San Vicente Fundación; profesor asistente, Cirugía general, Universidad de Antioquia, Medellín, Colombia.

3 Médico, especialista en Cirugía oncológica; cirujano asistencial, Hospital Pablo Tobón Uribe; profesor asistente, Cirugía general, Universidad de Antioquia, Medellín, Colombia.

4 MD, MSc., especialista en Cirugía general, magíster en Epidemiología Clínica, profesor titular, Cirugía general, Universidad de Antioquia, Medellín, Colombia.
\end{abstract}

\section{Resumen}

Introducción. El manejo de pacientes con cáncer gástrico está determinado por el estadio preoperatorio y requiere de una estrategia multidisciplinaria. La cirugía radical, especialmente en pacientes con estadios tempranos, es potencialmente curativa. El abordaje por vía laparoscópica ofrece ventajas sobre la vía abierta, sin embargo, en nuestro medio no hay información en cuanto a resultados oncológicos y posoperatorios tempranos.

Métodos. Estudio retrospectivo, descriptivo, de tipo transversal, en pacientes con cáncer gástrico llevados a gastrectomía laparoscópica con intención curativa, entre el 2014 y el 2019, en tres instituciones de la ciudad de Medellín. Se analizaron los datos demográficos, los resultados posoperatorios y oncológicos a corto plazo.

Resultados. Se incluyeron 75 pacientes sometidos a gastrectomía laparoscópica. La mediana de edad fue de 64 años y el estadio más frecuente fue el III. La gastrectomía fue subtotal en 50 pacientes $(66,7 \%)$ y total en 25 pacientes (33,3\%). Se hizo disección linfática D2 en 73 pacientes (97,3\%) con una mediana en el recuento ganglionar de 27. La tasa de resección R0 fue de 97,3\%. La mediana de estancia hospitalaria fue de seis días. La tasa de complicaciones mayores fue del $20 \%$ y la mortalidad a 90 días fue del $4 \%$.

Fecha de recibido: 19/06/2020 - Fecha de aceptación: 19/08/2020

Correspondencia: Paula Andrea Jurado Muñoz, Carrera 51D N 62 - 69, Medellín, Colombia. Teléfono: 3187955652 - (574) 2192469 Correo electrónico: paulajurado@gmail.com

Citar como: Jurado-Muñoz PA, Bustamante-Múnera RH, Toro-Vásquez JP, Correa-Cote JC, Morales-Uribe CH. Resultados tempranos en pacientes con cáncer gástrico sometidos a gastrectomía laparoscópica con intención curativa. Rev Colomb Cir. 2021;36:74-82. https://doi.org/10.30944/20117582.703

Este es un artículo de acceso abierto bajo una Licencia Creative Commons - BY-NC-ND https://creativecommons.org/licenses/by-ncnd/4.0/deed.es 
Discusión. La calidad oncológica de la gastrectomía laparoscópica fue adecuada y cumple con las recomendaciones de las guías internacionales. Si bien la morbilidad sigue siendo alta, tiene una tasa de sobrevida del $96 \%$ a 90 días.

Palabras clave: gastrectomía radical laparoscópica; cáncer gástrico; resultado oncológico; complicaciones posoperatorias.

\begin{abstract}
Introduction. The management of patients with gastric cancer is determined by the preoperative stage and requires a multidisciplinary strategy. Radical surgery, especially in patients with early stages, is potentially curative. The laparoscopic approach offers advantages over the open approach, however, in our setting there is no information regarding oncological and early postoperative results.
\end{abstract}

Methods. Retrospective, descriptive, cross-sectional study in patients with gastric cancer who underwent laparoscopic gastrectomy with curative intent, between 2014 and 2019, in three institutions in the city of Medellín. Demographic data, postoperative and short-term oncological results were analyzed.

Results. Seventy-five patients undergoing laparoscopic gastrectomy were included. The median age was 64 years and the most frequent stage was III. Gastrectomy was subtotal in 50 patients (66.7\%) and total in 25 patients (33.3\%). D2 lymphatic dissection was performed in 73 patients (97.3\%) with a median lymph node count of 27. The R0 resection rate was $97.3 \%$. The median hospital stay was six days. The major complication rate was $20 \%$ and the 90 -day mortality was $4 \%$.

Discussion. The oncological quality of the laparoscopic gastrectomy was adequate and complies with the recommendations of international guidelines. Although morbidity remains high, it has a $96 \%$ survival rate at 90 days.

Keywords: radical laparoscopic gastrectomy; gastric cancer; oncologic result; postoperative complications.

\section{Introducción}

El cáncer gástrico es uno de los cánceres más comunes a nivel mundial, afectando principalmente a países en vía de desarrollo con más del $70 \%$ de los casos. En Colombia, la incidencia es de 12,8 por 100.000 habitantes, ocupando el cuarto lugar en frecuencia y la primera causa de muerte por cáncer ${ }^{1}$. El tratamiento de pacientes con cáncer gástrico se define de acuerdo con el estadio de la enfermedad, dado por la clasificación clínica del TNM e incluye diferentes modalidades como la resección endoscópica, resección quirúrgica, radioterapia y quimioterapia.

En pacientes con enfermedad local o locoregional, el manejo quirúrgico con intención curativa sigue siendo el pilar fundamental del tratamiento asociado a la terapia perioperatoria. Cuando está indicado, se debe realizar una gastrectomía radical con un margen proximal negativo macroscópicamente de $5 \mathrm{~cm}$ o de $8 \mathrm{~cm}$ en casos de adenocarcino- ma de tipo difuso, además de una disección linfática D2 resecando al menos 16 ganglios que permitan una estadificación patológica confiable ${ }^{2-5}$. Se han demostrado ventajas del abordaje mínimamente invasivo sobre el abordaje abierto, en relación a menor pérdida sanguínea, dolor y estancia hospitalaria, así como tiempo de recuperación más corto, con unos resultados operatorios y oncológicos equivalentes, en manos de cirujanos experimentados ${ }^{4,6}$.

El objetivo de este trabajo fue conocer los resultados posoperatorios tempranos y la calidad oncológica de la gastrectomía radical laparoscópica en pacientes con cáncer gástrico tratados en nuestra ciudad.

\section{Métodos}

\section{Selección de pacientes}

Se llevó a cabo un estudio retrospectivo, descriptivo, de tipo transversal, en el que se incluyeron pacientes mayores de 18 años con diagnóstico de 
adenocarcinoma gástrico, llevados a gastrectomía radical laparoscópica con intención curativa en el tres instituciones de Medellín, Colombia, entre enero de 2014 y septiembre de 2019 . Se excluyeron pacientes intervenidos con intención paliativa y por vía abierta.

\section{Técnica quirúrgica}

La gastrectomía radical laparoscópica se realizó con una técnica de 5 puertos, iniciando con la omentectomía, seguido de la disección ganglionar D2 y la resección gástrica, que se efectuó con sutura mecánica, garantizando márgenes macroscópicos negativos de acuerdo con la localización y el tipo histológico del tumor. En algunos casos de tumores proximales se usó la endoscopia intraoperatoria para asegurar un margen adecuado.

Se finalizó con la reconstrucción del tracto digestivo en $\mathrm{Y}$ de Roux, con anastomosis gastro-yeyunal y yeyuno-yeyunal mediante sutura mecánica y la esófago-yeyunal con sutura manual, mecánica circular o lineal, según la preferencia del cirujano. Del mismo modo, la extracción de la pieza quirúrgica se hizo por una incisión de Pfannenstiel o ampliando uno de los puertos previa protección de la herida.

\section{Datos clínicos}

Se hizo una revisión retrospectiva de una base de datos recolectada de manera prospectiva. Los resultados primarios fueron los resultados oncológicos y postoperatorios tempranos (morbilidad, mortalidad y estancia hospitalaria). Las complicaciones tempranas, es decir las ocurridas en los primeros 30 días, se clasificaron según el sistema Clavien-Dindo, considerando menores las de grado I - II y mayores las de grado III - V. Los resultados oncológicos se consideraron adecuados cuando se obtuvieron márgenes de resección microscópicamente negativos y la resección de al menos 16 ganglios linfáticos.

\section{Análisis estadístico}

Se hizo un análisis descriptivo de las variables, presentando el porcentaje para las variables categóricas y mediana o media para las continuas, de acuerdo con su distribución.

\section{Resultados}

De un total de 125 pacientes llevados a gastrectomía laparoscópica entre enero de 2014 y septiembre de 2019, se excluyeron aquellos intervenidos con intención paliativa o por causas diferentes a adenocarcinoma gástrico, contando finalmente con un total de 75 pacientes para el estudio (figura 1). De ellos, 49 pacientes (65,3\%) fueron hombres, y la mediana de edad fue de 64 años (RIQ 55 - 74).

Según la clasificación de la octava edición de AJCC (American Joint Committee on Cancer), un

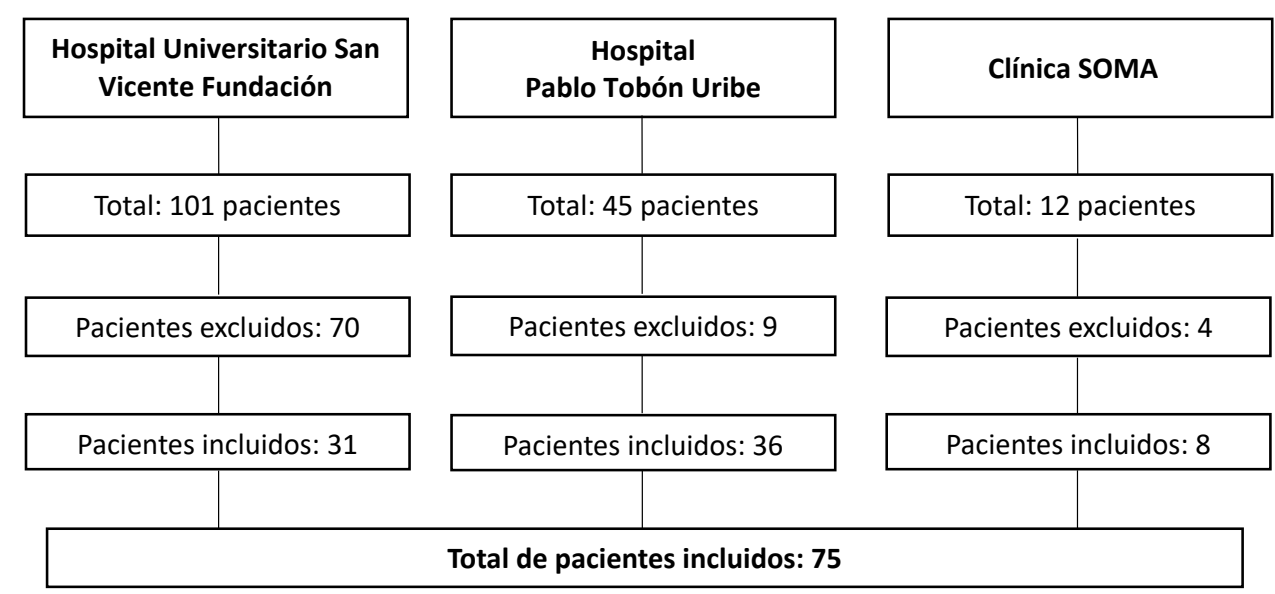

Figura 1. Pacientes llevados a gastrectomía con intención paliativa o gastrectomía por causa diferente a adenocarcinoma. 
paciente $(1,3 \%)$ tenía una enfermedad estadio $0,14(18,7 \%)$ estadio I, $23(30,7 \%)$ estadio II y 37 (49,3\%) estadio III. Solo tres pacientes (4\%) recibieron terapia neoadyuvante, en tanto que 43 pacientes $(57,3 \%)$ recibieron terapia adyuvante (tabla 1).

Se realizó gastrectomía subtotal en 50 pacientes $(66,7 \%)$ y disección ganglionar D2 en 73 pacientes $(97,3 \%)$. El procedimiento se terminó por vía laparoscópica en 74 pacientes $(98,7 \%)$ y en un paciente $(1,3 \%)$ se convirtió a cirugía abierta. El tiempo quirúrgico y el sangrado intraoperatorio alcanzaron una mediana de 240 minutos y 150 mililitros respectivamente, con necesidad de transfusión intraoperatoria en cuatro pacientes $(5,3 \%)$ (tabla 2$)$.

El tipo histológico intestinal se documentó en 38 pacientes $(50,7 \%)$, el difuso en $25(33,3 \%)$ y el mixto en siete $(9,3 \%)$. Se encontró un grado histológico bien diferenciado (G1) en 21 pacientes (28\%), moderadamente diferenciado (G2) en 19 $(25,3 \%)$ y mal diferenciado o indiferenciado (G3) en 30 (40\%). Los márgenes de resección fueron negativos en 73 pacientes $(97,3 \%)$ y la linfadenectomía tuvo una mediana de 27 ganglios resecados, con compromiso ganglionar por adenocarcinoma en 44 pacientes $(58,7 \%)$ (tabla 3$)$.

La mediana de la estancia hospitalaria fue de seis días. Se registró alguna morbilidad a 30 días en 23 pacientes $(30,7 \%)$, de ellos, 15 pacientes (20\%) presentaron complicaciones mayores y $8(10,7 \%)$ complicaciones menores, donde 8 $(10.6 \%)$ presentaron filtración de alguna de las anastomosis y $3(4 \%)$ presentaron fuga del muñón duodenal. La neumonía asociada al cuidado de la salud, al igual que la infección de tracto urinario, se documentaron cada una en un paciente $(1,3 \%)$. Doce pacientes $(16 \%)$ reingresaron antes de 30 días y 14 (18,6 \%) antes de 90 días. Requirieron una intervención adicional 14 pacientes (18,6\%), en 7 fue manejo quirúrgico, en 3 manejo percutáneos, en 3 manejo combinado quirúrgico y endoscópico y en uno manejo endoscópico. Se presentó mortalidad a 30 y 90 días en tres pacientes (4\%) (tablas 4 y 5).
Tabla 1. Características clínicas y patológicas de los pacientes $(n=75)$.

\begin{tabular}{|c|c|c|}
\hline Características & Frecuencia & $\%$ \\
\hline Edad (mediana) & 64 & RIQ 55-74 \\
\hline Hombres & 49 & 65,3 \\
\hline Antecedente de tabaquismo & 32 & 42,7 \\
\hline Síndrome pilórico & 16 & 21,3 \\
\hline \multicolumn{3}{|l|}{ Clasificación ASA } \\
\hline I & 1 & 1,3 \\
\hline II & 20 & 26,37 \\
\hline III & 49 & 65,3 \\
\hline IV & 5 & 6,7 \\
\hline \multicolumn{3}{|l|}{ pTNM } \\
\hline Tis & 1 & 1,3 \\
\hline $\mathrm{T} 1 \mathrm{a}$ & 5 & 6,7 \\
\hline $\mathrm{T} 1 \mathrm{~B}$ & 5 & 6,7 \\
\hline $\mathrm{T} 2$ & 8 & 10,7 \\
\hline T3 & 20 & 26,7 \\
\hline $\mathrm{T} 4 \mathrm{a}$ & 32 & 42,7 \\
\hline $\mathrm{T} 4 \mathrm{~b}$ & 1 & 1,3 \\
\hline No & 29 & 38,6 \\
\hline $\mathrm{N} 1$ & 16 & 21,3 \\
\hline N2 & 5 & 6,7 \\
\hline N3a & 15 & 20 \\
\hline N3b & 7 & 9,3 \\
\hline MO & 72 & 96 \\
\hline M1 & 0 & 0 \\
\hline \multicolumn{3}{|l|}{ ypTNM } \\
\hline T3 & 1 & 1,3 \\
\hline $\mathrm{T} 4 \mathrm{a}$ & 2 & 2,7 \\
\hline No & 2 & 2,7 \\
\hline N3b & 1 & 1,3 \\
\hline MO & 3 & 4 \\
\hline \multicolumn{3}{|l|}{ Estadio } \\
\hline 0 & 1 & 1,3 \\
\hline $\mathrm{IA}$ & 10 & 13,3 \\
\hline IB & 4 & 5,3 \\
\hline IIA & 7 & 9,3 \\
\hline IIB & 16 & 21,3 \\
\hline IIIA & 13 & 17,3 \\
\hline IIIB & 15 & 20 \\
\hline IIIC & 9 & 12 \\
\hline Neoadyuvancia & 3 & 4 \\
\hline \multicolumn{3}{|l|}{ Adyuvancia } \\
\hline $\mathrm{Si}$ & 43 & 57,3 \\
\hline Sin información & 4 & 5,3 \\
\hline
\end{tabular}

${ }^{*} \mathrm{RIQ}$ : rango intercuartílico 
Tabla 2. Resultados operatorios.

\begin{tabular}{lccc}
\hline \multicolumn{1}{c}{ Características } & $\begin{array}{c}\text { Total de pacientes } \\
\mathbf{n = 7 5}(\%)\end{array}$ & $\begin{array}{c}\text { Gastrectomía total } \\
\mathbf{n = 2 5 ( \% )}\end{array}$ & $\begin{array}{c}\text { Gastrectomía subtotal } \\
\mathbf{n = 5 0}(\mathbf{\%})\end{array}$ \\
\hline Tipo de cirugía & & $25(33,3)$ & $50(66,7)$ \\
Conversión a cirugía abierta & $1(1,3)$ & $1(4)$ & 0 \\
Linfadenectomía & & & \\
$\quad$ D1 & $2(2,7)$ & 0 & $2(4)$ \\
D2 & $73(97,3)$ & $25(100)$ & $50(96)$ \\
Tiempo quirúrgico & $240 \mathrm{~min}(\mathrm{RIQ} 198-300)$ & $259 \mathrm{~min}(\mathrm{RIQ} 219-312)$ & $220(\mathrm{RIQ} 183-300)$ \\
(mediana) & $150 \mathrm{ml}(\mathrm{RIQ} 50-200)$ & $100(\mathrm{RIQ} 50-225)$ & $150(50-200)$ \\
Sangrado quirúrgico (mediana) & $4(5,3)$ & $3(12)$ & $1(2)$ \\
Transfusión intraoperatoria & & &
\end{tabular}

Tabla 3. Resultados histopatológicos en los pacientes incluidos en el estudio $(n=75)$.

\begin{tabular}{lcc}
\hline Características & Frecuencia & $\%$ \\
\hline Histología & 38 & 50,7 \\
Intestinal & 25 & 33,3 \\
Difuso & 7 & 9,3 \\
Mixto & 1 & 1,3 \\
Adenoescamaso & 2 & 2,7 \\
Linfoepitelial & 1 & 1,3 \\
$\quad$ Mucinoso invasor & 18 & 24 \\
Células en anillo de sello & & \\
Grado histológico & 21 & 28 \\
$\quad$ Bien diferenciado & 19 & 25,3 \\
Moderadamente diferenciado & 30 & 40 \\
Mal diferenciado/Indiferenciado & 5 & 6,7 \\
Sin información & & 0 \\
Márgenes de resección comprometidos & 0 & 0 \\
Proximal & 0 & 2,6 \\
Distal & 2 & RIQ 16-40 \\
Proximal y distal & & $\mathrm{RIQ} 0-9$ \\
Recuento ganglionar & 27 & \\
Ganglios totales (mediana) & 1 & \\
Ganglios positivos (mediana) & &
\end{tabular}

Tabla 4. Resultados postoperatorios tempranos.

\begin{tabular}{|c|c|c|c|}
\hline Características & $\begin{array}{c}\text { Total de pacientes } \\
n=75(\%)\end{array}$ & $\begin{array}{c}\text { Gastrectomía total } \\
n=25(\%)\end{array}$ & $\begin{array}{c}\text { Gastrectomía subtotal } \\
n=50(\%)\end{array}$ \\
\hline Estancia hospitalaria en días (mediana) & $6(\mathrm{RIQ} 4-10)$ & 6 (RIQ 4-11) & 5.5 (RIQ 4-8) \\
\hline \multicolumn{4}{|l|}{ Complicaciones postoperatorias } \\
\hline Menores & $8(10,7)$ & $4(16)$ & $4(8)$ \\
\hline Mayores & $15(20)$ & $5(20)$ & $10(20)$ \\
\hline Re intervención & $14(18,6)$ & $5(20)$ & $9(18)$ \\
\hline \multicolumn{4}{|l|}{ Reingresos } \\
\hline 30 días postoperatorios & $12(16)$ & $3(12)$ & $9(18)$ \\
\hline 90 días postoperatorios & $14(18,6)$ & $4(16)$ & $10(20)$ \\
\hline \multicolumn{4}{|l|}{ Mortalidad } \\
\hline 30 días postoperatorios & $3(4)$ & $1(4)$ & $2(4)$ \\
\hline 90 días postoperatorios & $3(4)$ & $1(4)$ & $2(4)$ \\
\hline
\end{tabular}


Tabla 5. Complicaciones.

\begin{tabular}{lcc}
\hline Características & $\begin{array}{c}\text { Pacientes } \\
\mathbf{n = 7 5}\end{array}$ & $\%$ \\
\hline Total de complicaciones & 26 & \\
Total de pacientes complicados & 23 & 30,7 \\
Infección del sitio operatorio & & \\
$\quad$ Incisional superficial & 0 & 0 \\
$\quad$ Incisional profunda & 1 & 1,3 \\
$\quad$ Órgano-espacio & 2 & 2,7 \\
Filtración de la anastomosis & & \\
$\quad$ Esófago-yeyunal & 5 & 6,6 \\
Gastro-yeyunal & 3 & 4 \\
Yeyuno-yeyunal & 0 & 0 \\
Fugas del muñón duodenal & 3 & 4 \\
Neumonía asociada al cuidado de la salud & 1 & 1,3 \\
Infección de tracto urinario & 1 & 1,3 \\
Otras & & \\
Necrosis del remanente gástrico & 3 & 4 \\
Pseudoaneurisma arteria hepática & 1 & 1,3 \\
Pancreatitis posoperatoria & 1 & 1,3 \\
Fístula quilosa & 1 & 1,3 \\
Estenosis yeyuno-yeyuno anastomosis & 1 & 1,3 \\
Trombosis venosa profunda & 1 & 1,3 \\
Hernia hiatal aguda & 1 & 1,3 \\
Sospecha de isquemia intestinal & 1 & 1,3 \\
\hline
\end{tabular}

\section{Discusión}

La técnica mínimamente invasiva es la preferida por diferentes grupos quirúrgicos en varios centros a nivel mundial para el tratamiento del cáncer gástrico en pacientes bien seleccionados, documentándose diferentes ventajas, además de resultados postoperatorios y oncológicos equivalentes a la técnica abierta ${ }^{6}$.

En general, las características clínicas de los pacientes incluidos en el presente estudio son similares a las de los pacientes de otras regiones a nivel mundial ${ }^{7-11}$, con predominio de pacientes en la séptima década de la vida y género masculino en la mayoría de los casos. Cerca de la mitad de los pacientes fueron clasificados como ASA III, indicando la presencia de una enfermedad sistémica grave, asociada al antecedente de tabaquismo, el cual es un factor de riesgo conocido para el desarrollo de cáncer gástrico ${ }^{2}$.

El estadio más frecuente fue el III, seguido del estadio II con $37,3 \%$ y $30 \%$ de los casos respectivamente, indicando mayor proporción de pacientes con enfermedad locorregional, acorde a los reportes de estudios locales y americanos ${ }^{11,12}$. En contraste, en países orientales predominan los estadios I y II ${ }^{10,13,14}$, posiblemente debido a la existencia de programas de tamización. Se encontró que sólo el $4 \%$ de los pacientes recibieron terapia neoadyuvante, lo cual refleja la subutilización de este recurso cuando está indicado.

El tipo histológico intestinal y grado de diferenciación tumoral G3 son los hallazgos histológicos más frecuentes ${ }^{9,15}$, lo cual también fue documentado en el presente estudio con 50,7 \% de los casos con subtipo intestinal, $40 \%$ de tumores G3 y $24 \%$ con células en anillo de sello.

Independientemente del abordaje y el tipo de cirugía, los objetivos quirúrgicos en el paciente con cáncer gástrico, incluyen la resección completa del tumor con márgenes microscópicos negativos y disección ganglionar de al menos 16 ganglios ${ }^{4,6}$. Tal como se ha documentado en estudios americanos y asiáticos $8,11,14,16,17$, el tipo de cirugía más frecuente en el presente estudio fue la gastrectomía subtotal con un $66,7 \%$, alcanzando una resección R0 en el 97,3 \% de los pacientes y R1 en el 2,7 \% restante. La linfadenectomía D1 se realizó en dos pacientes, uno de 90 años con múltiples comorbilidades y otro con una enfermedad estadio 0 . A los 73 pacientes restantes, se les realizó una linfadenectomía D2 con una mediana de 27 ganglios resecados y más del $25 \%$ de los casos con recuentos mayores a 16, logrando de esta manera el cumplimiento de las recomendaciones de las guías internacionales ${ }^{2,5}$.

Sólo uno de los pacientes requirió conversión a cirugía abierta, por compromiso tumoral de la arteria esplénica, documentado durante el intraoperatorio. Esto corresponde a una tasa de conversión del 1,3\%, siendo menor a la reportado en la literatura, que menciona valores alrededor de $6,4 \%{ }^{7,13,18}$. La mediana global para el tiempo quirúrgico y el sangrado intraoperatorio fueron de 240 minutos y 150 mililitros respectivamente, comparables con lo informado en diferentes estudios $7,8,16,17$. La estancia hospitalaria fue similar a la reportada previamente ${ }^{7,14,16,19}$ con una mediana de seis días.

La mayoría de estudios publicados sobre el abordaje quirúrgico en cáncer gástrico son de 
gastrectomías laparoscópicas subtotales realizados en países asiáticos, donde tienen una mayor incidencia de la enfermedad y una mayor proporción de estadios tempranos en comparación con países occidentales. Es por esto que los estudios occidentales como el nuestro, incluyen pacientes con un número limitado y en estadios más avanzados. Lo anterior posiblemente explica la diferencia entre las tasas de morbimortalidad entre países asiáticos y el resto del mundo, basado en una intervención en estadios más tempranos y en un mayor volumen de pacientes, lo cual se traduce en una experiencia quirúrgica superior.

En el presente estudio, la morbilidad general fue del $30 \%$, considerándose complicaciones mayores (Clavien-Dindo III - V) en $20 \%$ de los pacientes, valores comparables con los informados en estudios americanos como el de Kaitlyn y Guzmán, que incluyeron pacientes llevados a gastrectomía radical laparoscópica tanto total como subtotal ${ }^{18,20}$ con una morbilidad del $30 \%$ y complicaciones mayores en el $14 \%$. En general, la complicación más frecuente con un 10,6\% fue la filtración de alguna de las anastomosis, presente en ocho pacientes, cinco con filtración de la anastomosis esófago-yeyunal $\mathrm{y}$ tres de la gastro-yeyunal.

Comparando con otros estudios, la tasa de filtración de las anastomosis fue ligeramente mayor y la tasa de fuga del muñón duodenal fue muy similar 7,8,15,18,20. La infección de tracto urinario y la neumonía asociada al cuidado de la salud se presentaron en el 1,3\% de los casos, esta última con una menor incidencia a la reportada en la literatura ${ }^{7,21,22}$. Se presentaron tres infecciones de sitio operatorio ( $4 \%$ ), valor que está dentro de lo reportado en la literatura y confirma una de las ventajas del abordaje mínimamente invasivo ${ }^{7-9,15,18,22}$. Tres pacientes murieron dentro de los 30 días posoperatorios, uno por sepsis abdominal debida a filtración de la anastomosis esófago-yeyunal, otro por filtración del muñón duodenal y el último por posible isquemia intestinal, la cual no se pudo confirmar por decisión familiar de limitar el esfuerzo terapéutico.

Como limitaciones del estudio, debemos mencionar la naturaleza retrospectiva, y que, a pesar de tomarse las medidas necesarias para tener una buena calidad en los resultados, se puede presen- tar omisión de los mismos, relacionada con la falta de control de sesgos e información parcial de algunas de las variables estudiadas. Otro factor es el tamaño limitado de la muestra, posiblemente por la diferencia de los períodos incluidos en las distintas instituciones y por el sistema de codificación de la patología y las intervenciones incluidas en cada una de ellas.

La falta de estudios locales sobre resultados tempranos en cirugía radical laparoscópica en cáncer gástrico limita una adecuada interpretación de la tasa de morbimortalidad obtenida. Sin embargo, este trabajo permitió conocer la epidemiología de los pacientes manejados en nuestras instituciones, la calidad oncológica de la cirugía e identificar los principales resultados posoperatorios tempranos. Este trabajo plantea a su vez, posibles puntos de intervención como la estandarización de la técnica quirúrgica y la optimización preoperatoria de los pacientes para obtener mejores resultados clínicos. Asimismo, es el punto de partida para diseñar estudios prospectivos con mayor número de pacientes y seguimiento a largo plazo, que permitan obtener una información más completa y precisa del desempeño de la cirugía laparoscópica en pacientes con cáncer gástrico.

\section{Conclusiones}

El presente estudio demuestra que la calidad oncológica de la gastrectomía laparoscópica con intención curativa en pacientes con cáncer gástrico es adecuada y cumple las recomendaciones de las guías internacionales. Este abordaje permitió una resección $\mathrm{R} 0$ y disección ganglionar tipo D2 en el $97 \%$ de los pacientes. A pesar de que la morbilidad general es alta, es comparable con estudios americanos. La intervención se asoció a una tasa baja de transfusiones intraoperatorias, a una tasa baja de complicaciones pulmonares y a una estancia hospitalaria corta. La supervivencia a 90 días fue del $96 \%$.

\section{Cumplimiento de normas éticas}

Consentimiento informado: Por tratarse de un estudio retrospectivo de revisión de historias clínicas, este es un estudio sin riesgo de acuerdo con lo definido en la 
resolución 008430 de 1993 del Ministerio de Salud de Colombia, por lo que no se requirió consentimiento informado. Se obtuvo el aval de los comités de investigación y de ética de la Universidad y de las instituciones participantes.

Conflictos de interés: Ninguno declarado por los autores.

Financiación: Autofinanciado.

Contribución de los autores: Concepción y diseño del estudio, adquisición, análisis e interpretación de datos y redacción del manuscrito: Paula Andrea Jurado Muñoz, Robin Hernando Bustamante-Múnera.

Concepción y diseño del estudio, análisis e interpretación de datos, revisión crítica del manuscrito: Juan Pablo ToroVásquez, Juan Camilo Correa-Cote, Carlos Hernando Morales-Uribe.

\section{Referencias}

1. Bray F, Ferlay J, Soerjomataram I, Siegel RL, Torre LA, Jemal A. Global cancer statistics 2018: GLOBOCAN estimates of incidence and mortality worldwide for 36 cancers in 185 countries. CA Cancer J Clin. 2018;68:394-424. https://doi.org/10.3322/caac.21492

2. Smyth EC, Verheij M, Allum W, Cunningham D, Cervantes A, Arnold D, et al. Gastric cancer: ESMO clinical practice guidelines for diagnosis, treatment and follow-up. Ann Oncol. 2016;27:v38-49.

https://doi.org/10.1093/annonc/mdw350.

3. Ajani JA, In H, Sano T, Gaspar LE, Erasmus JJ, Tang LH, et al. Stomach. In: AJCC Cancer Staging Manual, 8th, Amin MB. (Ed), AJCC, Chicago. 2017:203.

4. Gholami S, Cassidy MR, Strong VE. Minimally invasive surgical approaches to gastric resection. Surg Clin North Am. 2017;97:249-64. https://doi.org/10.1016/j.suc.2016.11.003.

5. Ajani Aj, D’Amico TA, Bentrem DJ, Chao j, Das P, Denlinger CS, et al. . National Comprehensive Cancer Network (NCCN). NCCN clinical practice guidelines in oncology. Gastric Cancer Version 4.2019. December 20, 2019.

6. Costantino CL, Mullen JT. Minimally invasive gastric cancer surgery. Surg Oncol Clin N Am. 2019;28:201-13. https://doi.org/10.1016/j.soc.2018.11.007

7. Hu Y, Huang C, Sun Y, Su X, Cao H, Hu J, et al. Morbidity and mortality of laparoscopic versus open D2 distal gastrectomy for advanced gastric cancer: A randomized controlled trial. J Clin Oncol. 2016;34:1350-7. https://doi.org/10.1200/JC0.2015.63.7215.

8. Kinoshita T, Uyama I, Terashima M, Noshiro H, Nagai E, Obama K, et al. Long-term outcomes of laparosco- pic versus open surgery for clinical stage ii/iii gastric cancer: A Multicenter Cohort Study in Japan (LOC-A Study). Ann Surg. 2019;269:887-94.

https://doi.org/10.1097/SLA.0000000000002768.

9. Lee JH, Nam BH, Ryu KW, Ryu SY, Park YK, Kim S, et al. Comparison of outcomes after laparoscopy-assisted and open total gastrectomy for early gastric cancer. $\mathrm{Br}$ J Surg. 2015;102:1500-5. https://doi.org/10.1002/bjs.9902.

10. Kim HH, Han SU, Kim MC, Kim W, Lee HJ, Ryu SW, et al. Effect of laparoscopic distal gastrectomy vs open distal gastrectomy on long-term survival among patients with stage I gastric cancer: The KLASS-01 randomized clinical trial. JAMA Oncol. 2019;5:506-13.

https://doi.org/10.1001/jamaoncol.2018.6727.

11. Bernal Mesa JA, Dorado EAA. Impacto de la radicalidad quirúrgica en el manejo definitivo del cáncer gástrico. Estudio de dos instituciones en Medellín. Rev Colomb Gastroenterol. 2008;23:251-8.

12. Ecker BL, Datta J, McMillan MT, Poe SLC, Drebin JA, Fraker DL, et al. Minimally invasive gastrectomy for gastric adenocarcinoma in the United States: Utilization and short-term oncologic outcomes. J Surg Oncol. 2015;112:616-21. https://doi.org/10.1002/jso.24052

13. Yu J, Huang C, Sun Y, Su X, Cao H, Hu J, et al. Effect of laparoscopic vs open distal gastrectomy on 3-year disease-free survival in patients with locally advanced gastric cancer: The CLASS-01 randomized clinical trial. JAMA. 2019;321:1983-92. https://doi.org/10.1001/jama.2019.5359.

14. Sugita H, Kojima K, Inokuchi M, Kato K. Long-term outcomes of laparoscopic gastrectomy for gastric cancer. J Surg Res. 2015;193:190-5. https://doi.org/10.1007/s00464-014-3856-4.

15. Lin JX, Huang CM, Zheng CH, Li P, Xie JW, Wang J Bin. Surgical outcomes of 2041 consecutive laparoscopic gastrectomy procedures for gastric cancer: A large-scale case control study. PLoS One. 2015;10:1-11. https://doi.org/10.1371/journal.pone.0114948.

16. Cui M, Li Z, Xing J, Yao Z, Liu M, Chen L, et al. A prospective randomized clinical trial comparing D2 dissection in laparoscopic and open gastrectomy for gastric cancer. Med Oncol. 2015;32:1-7. https:doi:10.1007/s12032-015-0680-1.

17. Escalona PA, Báez VS, Pimentel MF, Calvo BA, Boza WC, Viñuela FE, et al. Gastrectomía laparoscópica en cancer gástrico TT. Rev Chil Cir. 2008;60:188-93. https://doi.org/10.4067/S0718-40262008000300004.

18. Kelly KJ, Selby L, Chou JF, Dukleska K, Capanu M, Coit DG, et al. Adenocarcinoma in the west : A case - control Study. Ann Surg Oncol. 2015;22:3590-6. https://doi.org/10.1245/s10434-015-4381-y. 
19. Zeng Y-K, Yang Z-L, Peng J-S, Lin H-S, Cai L. Laparoscopy-assisted versus open distal gastrectomy for early gastric cancer. Ann Surg. 2012;256:39-52.

https://doi.org/10.1097/SLA.0b013e3182583e2e.

20. Guzman EA, Pigazzi A, Lee B, Soriano PA, Nelson RA, Benjamin Paz I, et al. Totally laparoscopic gastric resection with extended lymphadenectomy for gastric adenocarcinoma. Ann Surg Oncol. 2009;16:2218-23. https://doi.org/10.1245/s10434-009-0508-3.

21. Higgins RM, Kubasiak JC, Jacobson RA, Janssen I, Myers JA, Millikan KW, et al. Outcomes and use of laparoscopic versus open gastric resection. J Soc Laparoendosc Surg. 2015;19:e2015.00095.

https://doi.org/10.4293/JSLS.2015.00095.

22. Lee HJ, Hyung WJ, Yang HK, Han SU, Park YK, An JY, et al. Short-term outcomes of a multicenter randomized controlled trial comparing laparoscopic distal gastrectomy with D2 lymphadenectomy to open distal gastrectomy for locally advanced gastric cancer (KLASS-02-RCT). Ann Surg. 2019;270:983-91. https://doi.org10.1097/SLA.0000000000003217 